\title{
COVALENTLY CROSS-LINKED HYALURONIC ACID/BSA/GELATINE HYDROGELS AS BETTER SURFACE FOR CELL CULTURE
}

\author{
Simon KLIMOVIC ${ }^{1}$, Jan PRIBYL ${ }^{1}$, Vladimir ROTREKL ${ }^{2,3}$, Šárka JELINKOVA ${ }^{2,3}$, Hana VICAROVA \\ ${ }^{1}$ CEITEC, Masaryk University, Brno, Czech Republic, EU, simon.klimovic@ceitec.muni.cz, \\ jan.pribyl@ceitec.muni.cz \\ ${ }^{2}$ Department of Biology, Faculty of Medicine, Masaryk University, Brno, Czech Republic, EU, \\ vrotrekl@med.muni.cz, sarka.jelinkova89@gmail.com \\ 3International Clinical Research Center (ICRC), St.Anne's University Hospital, Brno, Czech Republic, EU
}

https://doi.org/10.37904/nanocon.2020.3739

\begin{abstract}
The mechanical and biomechanical properties of the cell's extracellular matrix is a factor in their later development. In this regard, elastic biocompatible materials as a surface for cultivating cells are in recent years, becoming an exciting field of study. Hydrogels are a hydrophilic 3D network of synthetic polymers or biopolymers. They can have tunable mechanical properties, controllable degradability, or they can be designed for long-time release of treatments. Here, hydrogels from Hyaluronic acid (HA), Bovine serum albumin (BSA) and gelatin was prepared, via carbodiimide chemistry. Morphology and mechanical properties of these hydrogels was characterized via Atomic Force Microscopy (AFM). Young's modulus of HA/BSA and $\mathrm{HA} / \mathrm{BSA} / \mathrm{gel}$ were $124.7 \pm 15.9$ and $20.3 \pm 0.5 \mathrm{kPa}$, respectively. Mouse embryonic fibroblasts were then cultivated on hydrogels and on the glass surface as a reference. Mechanical and morphological properties of fibroblasts were examined by AFM and via phalloidin-TRITC staying. Relationship between the structure of cells and the type of surface was seen. Cells grown on glass were more flattened with an evenly linear actin structure. On hydrogels, fibroblasts were more star-shaped with non-linear actin structures and many filopodia. Furthermore, height of cells was higher was lower compared to cells on both types of hydrogels $(0.36 \mu \mathrm{m} v \mathrm{~s}$. $0.56 \mu \mathrm{m}$ vs. $1.03 \mu \mathrm{m}$ ). Young's modulus of cells on fibroblast was highest, which relates to the whole range of structural changes linked with surface stiffness. In conclusion, this type of hydrogel might be utilized in the future as a better surface material for cell cultivation.
\end{abstract}

Keywords: AFM, Hydrogel, Actin structure, hyaluronic acid

\section{INTRODUCTION}

Cells can recognize various cues by extracellular matrix (ECM), starting from various chemical signals and proteins interactions to ECM's mechanical properties. Each cell type favors a different set of these cues to work correctly. A classical approach consists in vitro cell culturing on plastic or glass surfaces. Although it is universally accepted as a standard cultivating surface, it does not mimic ECM in any way. Moreover, it has been reported that cells cultivating on flat surfaces can demonstrate the abnormal distribution of integrins or forced cell polarity [1]. It has also been shown that different mechanical properties of the growth surface affect morphology and differentiation, organelle organization, cell migration, or resistance to chemotherapy [1-4]. In recent years, there is an increasing need for a biocompatible scaffold for cell cultures with tunable mechanical properties and bioactivity. Hydrogels, 3D networks of synthetic or biopolymers with high water retention has proven useful in that regard [1,5-7]. To mimic ECM mechanical properties and high-water retention, it is ideal to synthesize hydrogels from biopolymers. As a major component of the extracellular matrix in various tissues, Hyaluronic acid (HA) comes to mind [8]. HA is anionic biopolymer, consisting of its structure $\mathrm{N}$ - 
acetylglucosamine and D-glucoronic acid repeating units. In the cell, HA forms long chains, partially stabilized by weaker bonds between them. To modulate mechanical properties such as stiffness and elasticity, HA must be further stabilized by cross-linking. Chemical modification of hyaluronic acid by carbodiimides (EDAC) has been often used to stabilized HA [5,9]. EDAC modify side-groups of HA and mediate ester bond formation between hydroxyl and carboxyl groups of HA. Other types of biopolymers or molecules can be mixed with HA and EDAC to get different mechanical properties, such as collagen [5] or gelatin [7]. Bovine serum albumin (BSA) by its similarity with human variant, is an interesting candidate as material for a hydrogel, with the capability to bind, retain and release a variety of molecules in a defined manner [10,11].

In this study, the hydrogel matrix from HA and BSA was prepared and characterized. For improved mechanical stability, 1-ethyl-3-(3-dimethylaminopropyl)-carbodiimide (EDAC), together with $\mathrm{N}$-hydroxysuccimid (NHS) was used as a cross-linking agent. Resulting hydrogels were characterized by Atomic Force Microscopy for topography and mechanical properties in various conditions (temperature, medium). Resulting hydrogels showed desirable mechanical properties; however, Young's Modulus (YM) was further modulated by the addition of gelatin into hydrogel mixture. To demonstrate different morphology of cells on softer substrates, mouse embryonic fibroblasts (MEF) were then cultured on hydrogels. Finally, the actin structures of cells by Phalloidin-TRITC staining was analyzed.

\section{METHODS}

\subsection{Materials}

BSA, EDAC, NHS, Gelatin and Phalloidin-TRITC were purchased from Sigma-Aldrich (St. Louis, MO, USA). Trimethylamine (TEA) and (3-Aminopropyl)triethoxysilane (APTES) used for silanization were purchased from Honeywell research chemicals (Charlotte, NC, USA). Hyaluronic acid ( $\mathrm{Mw}_{\mathrm{w}}=550 \mathrm{kDa}$ ) was kindly provided by Jan Víteček. HEPES was purchased from ROTH (Carl, Germany). Mitotically inactivated mouse embryonic fibroblasts (mEF) for cultivation were kindly provided to us by Vladimir Rotrekl research group, LF Masaryk University.

\subsection{Silanization of glass cover slips}

To immobilize hydrogels for AFM measurement, alkoxysilane molecules were introduced on glass slips surface. Firstly, glass slip was attached on the bottom of the petri dish (TPP, Trasadingen, Switzerland) and cleaned with chromic acid, deionized water, and ethanol. After that, dry and clean petri dish with a glass slip attached was put to a vacuum desiccator together with $50 \mu$ of TEA and $50 \mu$ l of APTES. Vacuum was applied for 10 minutes, and then samples were left in desiccator for 2 hours.

\subsection{Fabrication and cross-linking of HA/BSA hydrogels}

Hydrogel mixture was prepared from HA and BSA, with a weight ratio of 2:8, respectively. For $100 \mu \mathrm{l}$ of mixture, $50 \mu \mathrm{l}$ of HA $(32 \mathrm{mg} / \mathrm{ml})$ and $50 \mu \mathrm{l}$ of BSA $(128 \mathrm{mg} / \mathrm{ml})$ was added. Alternatively, $10 \mu \mathrm{l}$ of gelatin $(50 \mathrm{mg} / \mathrm{ml})$ was added at BSA's expense. After thorough mixing, $6 \mathrm{mg}$ of EDAC and $1 \mathrm{mg}$ NHS was put into a mixture. Resulted slurry was poured on a silanized glass coverslip. After two hours of polymerization at room temperature, $2 \mathrm{ml}$ of HEPES (50 mM) was added and hydrogels were left to swell overnight in $5{ }^{\circ} \mathrm{C}$.

\subsection{Characterization of topography and mechanical properties by AFM}

For topography measurements, "Imaging in contact" mode on Atomic Force Microscope (JPK NanoWizard 3, Bruker, Billerica, MA, USA) was used with HYDRA-2N-100N cantilever (AppNano, Mountain View, CA, USA). Imaged area was $10 \times 10 \mu \mathrm{m}$ with setpoint: $100 \mathrm{pN}$, iGain: 50, PGain: 0.006, Z range: $15 \mu \mathrm{m}$, and Scan rate: $1 \mathrm{~Hz}$. For the measurement of mechanical properties, "Force Mapping" mode with setpoint $0.5 \mathrm{nN}$, relative setpoint: $1 \mathrm{nN}$, IGain: $70 \mathrm{~Hz}$, PGain: 0.002 and extended time: $0.5 \mathrm{~s}$, was used. Lastly we "Quantitative imaging" mode for topography images was used. The temperature of samples was maintained at $37^{\circ} \mathrm{C}$ by Petri Dish Heater (Bruker, Billerica, MA, USA). 
Force maps acquired were processed in JPK Data Processing 5 software using the Sneddon-Hertz model. All other processing of AFM images was done in Gwydion software [12].

\subsection{Cultivation of cells on hydrogels and phalloidin staining}

Frozen mitotically inactivated mouse embryonic cells were thawed in a water bath $\left(37^{\circ} \mathrm{C}\right)$ and then transferred into $15 \mathrm{ml}$ tube. After that $12 \mathrm{ml}$ of cold mEF medium ( $86 \%$ KnockOut Dulbecco modified eagle medium, 10 $\%$ fetal bovine serum, $1 \%$ L-glutamine, $1 \%$ penicillin/streptomycin, $1 \%$ nonessential amino acids, $0,1 \mathrm{mM} \mathrm{2-}$ mercaptoethanol) was poured into a tube, which was then centrifuge $200 \mathrm{~g} / 5 \mathrm{~min}$. Medium above the cell pellet was removed and warm $\mathrm{mEF}$ medium $\left(37^{\circ} \mathrm{C}\right)$ was added. The resulting cell suspension was divided between hydrogels samples.

Cells were stained after two days of cultivation. Firstly, mEF medium was removed, cells were washed with PBS buffer and then fixated with 3.7\% paraformaldehyde in PBS for 5 minutes at room temperature. Samples were washed with PBS $3 x$ times and incubated with $0.1 \%$ Triton-X in PBS for 10 minutes and washed with PBS again. After that $200 \mu \mathrm{l}$ of phalloidin-TRITC was added for 60 minutes, washed in PBS, and $200 \mu \mathrm{l}$ of 0.1 $\%$ DAPI were added. Finally, samples were washed $2 x$ in PBS, dried and stored for further measurement.

The confocal microscope (LSM 700, Zeiss, Oberkochen, Germany) was used for imaging phalloidin and DAPI staining. Images were further processed in ImageJ software [13].

\section{RESULTS AND DISCUSSION}

3.1. Optimization and characterization of hydrogels by AFMIn the process of optimization HA/BSA hydrogel system, two sources of HA - Hyaluronic acid sodium salt and high molecular crosslinked Hyalobarrier gel, was tried. Latter mentioned source could not be sufficiently polymerized. Ester bond formation during EDC/NHS reaction occurs on hydroxyl and carboxyl groups of HA and BSA.

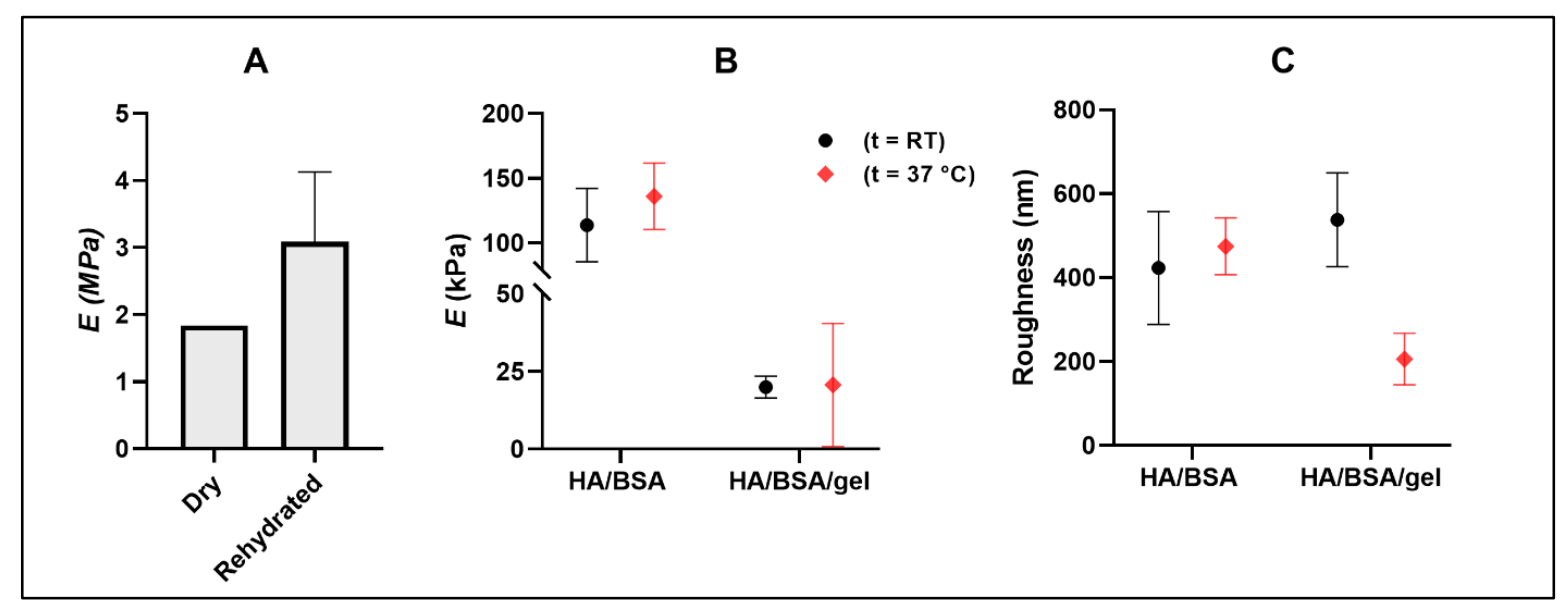

Figure 1 - Results from optimization of hydrogel preparation process. A - Difference between dry and rehydrated hydrogel, where we can see elevated YM of latter mentioned. B, C - Results of YM and

Roughness of $\mathrm{HA} / \mathrm{BSA}$ and $\mathrm{HA} / \mathrm{BSA} /$ Gelatine hydrogels in room temperature (RT) and $37^{\circ} \mathrm{C}$.

3.2. Hyalobarrier gel comes from production already partially polymerized and It probably either lacks active groups, or they are not available for the reaction. For further experiments, HA sodium salt was used. Mechanical properties of hydrogels without gelatin were tried first. During Force mapping, the cantilever frequently lost contact with the surface, and stiffness values were in units of MPa (Figure1A - Dry). High stiffness might have been caused by collapsing of hydrogel structure under AFM cantilever, which was previously shown $[14,15]$. Findings in previous study with alginate hydrogels suggested, that drying out and rehydrating of hydrogels can stabilize the structure. [16]. Therefore, same approach was utilized, 
which a partial success. While the cantilever was no longer losing contact with hydrogel, the stiffness was even more elevated. In figure 1A we can see YM for both dry and rehydrated hydrogels. It was concluded that hydrogel, after drying out, could not be rehydrated well enough, which on the one hand, might have caused better strength, but on the other hand, higher stiffness. Therefore, preparation process was optimized by letting hydrogels polymerized at room temperature with addition of HEPES buffer after two hours. Figure 1B shows YM of HA/BSA and HA/BSA/Gelatin hydrogels in room temperature and in $37{ }^{\circ} \mathrm{C}$. In previous studies, it has been stated that rotation around linkages in structure of HA are easier, with increasing temperature [17], causing better flexibility and, therefore, lower stiffness. Yet, this effect was not seen in our experiments. The addition of gelatin into a hydrogel mixture had an effect on YM (124.7 vs. 20.32, $P<0,0001)$. Roughness values did not differ significantly (Figure 1C). Figure 2 shows representative topography and YM AFM maps of HA/BSA and $\mathrm{HA} / \mathrm{BSA} / \mathrm{Gelatin}$ hydrogels. For cell cultivation, both HA/BSA and HA/BSA/gelatin hydrogels were chosen.

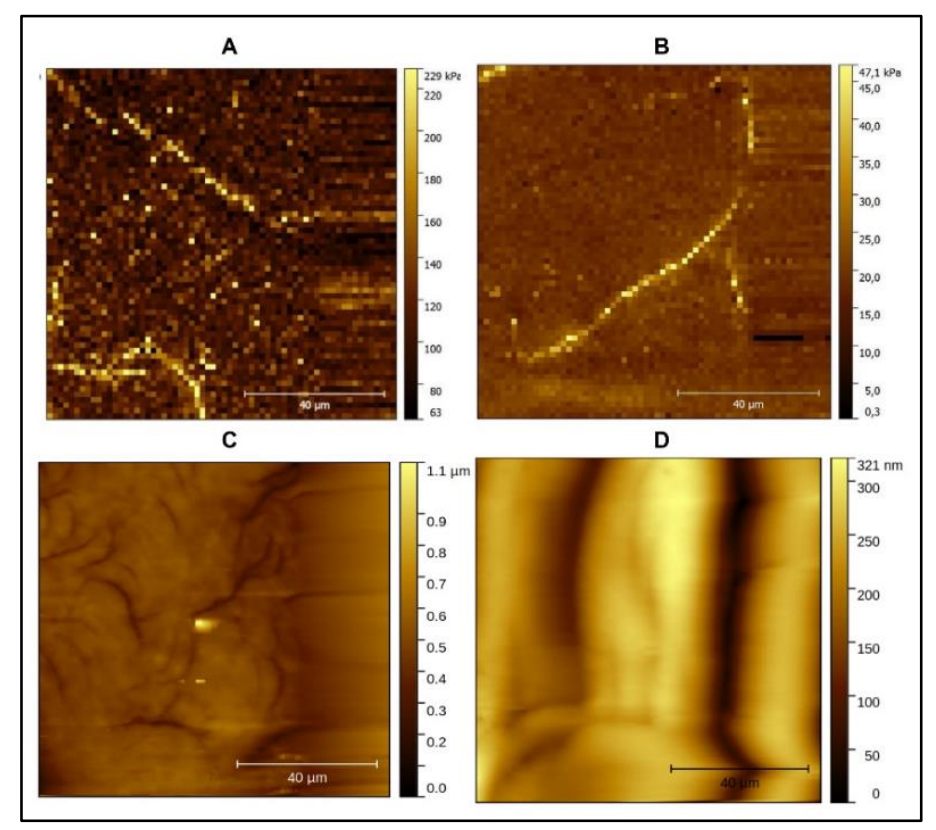

Figure 2 - Representative AFM maps of Young's Modulus and topography of HA/BSA hydrogel in RT (1A,

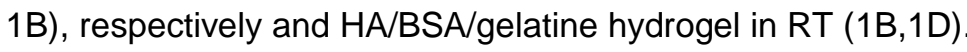

\subsection{Cultivation of cells and actin structures}

The primary aim of this experiment was to determine if and how cells will grow on hydrogels. Thus, Mouse embryonic fibroblast on HA/BSA, HA/BSA/gelatin hydrogels, and on glass for reference, were cultivated. The structure of cells was evaluated via actin staining. Topography and mechanical properties were measured by AFM. In Figure 4, pictures from confocal microscopy, actin structures via phalloidin-TRITC staining, and topography via AFM for the sample with reference surface, can be seen. Fibroblasts were most evenly grown on the glass. Actin structures and filopodia were mostly linear. On the other hand, cells on hydrogels were more irregular, often star-shaped, with more prominent filopodia and lamellipodia (arrows in 4E, H). Atomic Force microscopy was used to obtain topography images and mechanical properties of cells. Figure 3A shows that cell stiffness was highest on glass surface and dropped on hydrogels surfaces This results suggests that there is influence of cultivating surface on cell stiffness, firstly in the sense that the object with the same stiffness would appear softer on softer surfaces and vice versa, but secondly, due to changes in cellular structure, due stiffness of the surface. These changes in cellular structure are visible from topography images and actin structures, where cells are growing in a different manner, which is changing mechanical properties. 
Fibroblasts on glass were flatter (Figure 4C), and height was increasing with decreasing stiffness of surface. This trend is visible in Figure 3B.

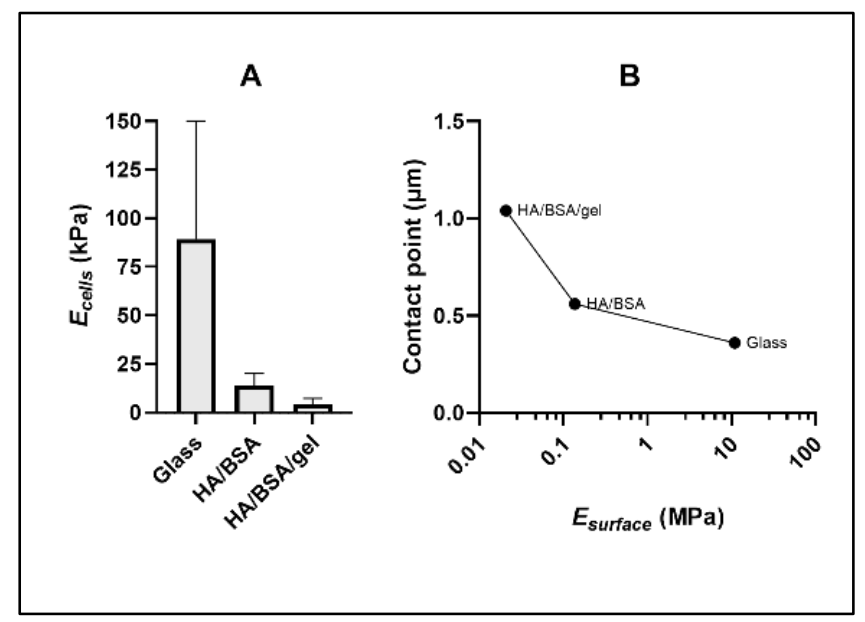

Figure 3 - A - Young's Modulus of cells was highest in glass sample and lowest in both hydrogels $(89.3 \pm$ 60.7 vs. $14.0 \pm 5.9$ vs $4.3 \pm 3.7$ ). B - Relationship between contact point of AFM cantilever in $\mu \mathrm{m}$ and Young's Modulus of surface. In other words, cells are more likely to flattened on stiff surfaces then on soft surfaces.

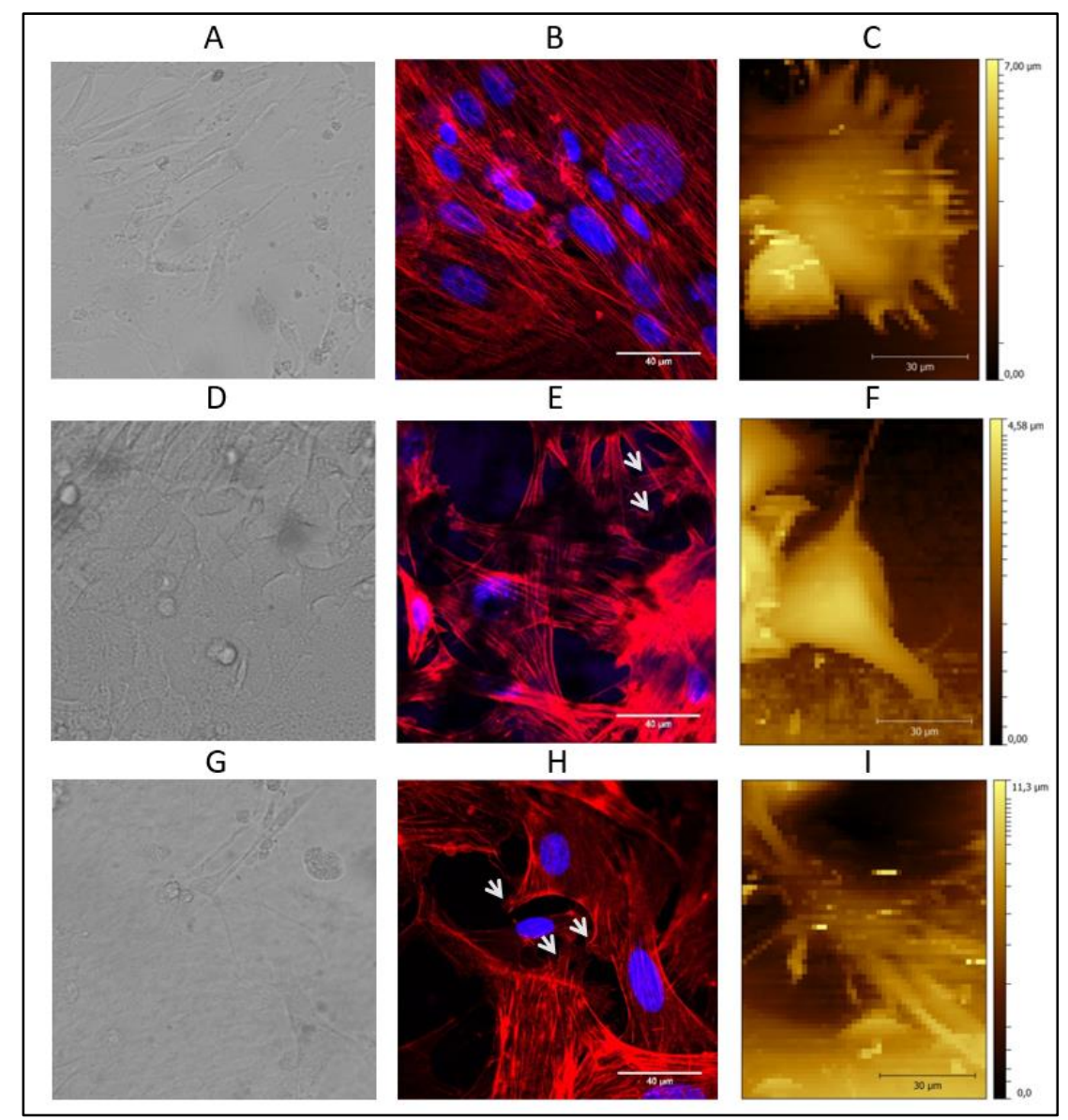

Figure 4 - Representative images of cells in confocal microscopy, actin structures and AFM topography. A-C - cells on glass; D-F - cells on HA/BSA hydrogel; G-I - cells on HA/BSA/gel hydrogel. (arrows represents filopodia and lamellipodia) 


\section{CONCLUSION}

In this study, hydrogels from hyaluronan, bovine serum albumin, and gelatin was prepared, using carbodiimide chemistry for cross-linking. Hydrogels were characterized by Atomic Force Microscopy, and stiffness values were calculated via the Hertz-Sneddon model from force curves. Values of Young's modulus were $124.7 \pm$ 15.9 and $20.3 \pm 0.5 \mathrm{kPa}$, for hydrogels with and without gelatin, respectively. Mouse embryonic fibroblasts were cultivated on both types of prepared scaffolds and on glass surface as a reference. The structure of cells was examined via confocal microscopy, Atomic Force Microscopy and actin structure was shown via staying with phalloidin conjugated with tetramethylrhodamine. Results showed great differences in cellular structure and morphology depending on surface type. Gupta et al. showed a connection between the stiffness of the surface and structure of actin filaments in a cell [18]. Cells on rigid surfaces tend to grow bigger are with linear actin structures and vice versa. Our findings correspond with the results of this study. In conclusion, we showed that this type of hydrogel can be used as a cultivation surface. Moreover, cells on these hydrogels are growing in a more physiologically manner than ones on standard surfaces such as glass.

\section{ACKNOWLEDGEMENTS}

CIISB, Instruct-CZ Centre of Instruct-ERIC EU consortium, funded by MEYS CR infrastructure project LM2018127, is gratefully acknowledged for the financial support of the measurements at the CF Nanobiotechnology. The work was also supported by the European Regional Development Fund Project ENOCH (No. CZ.02.1.01/0.0/0.0/16_019/0000868)

\section{REFERENCES}

[1] CALIARI, S.R., BURDICK, J.A.. A practical guide to hydrogels for cell culture. Nature Methods. 2016, vol. 13, no. 5, pp. 405-414.

[2] BALESTRINI, J.L., CHAUDHRY, S., SARRAZY, V., et al. The mechanical memory of lung myofibroblasts. Integrative Biology. 2012, vol. 4, no. 4, pp. 410-421.

[3] EVEN-RAM, S., YAMADA, K.M. Cell migration in 3D matrix. Current Opinion in Cell Biology. 2005, vol. 17, no. 5, pp. 524-532.

[4] NGUYEN, T.V., SLEIMAN, M., MORIARTY, T., et al. Sorafenib resistance and JNK signaling in carcinoma during extracellular matrix stiffening. Biomaterials. 2014, vol. 35, no. 22, pp. 5749-5759.

[5] PARK, S.-N., PARK, J.-C., KIM, H.O., et al. Characterization of porous collagen/hyaluronic acid scaffold modified by 1-ethyl-3-(3-dimethylaminopropyl)carbodiimide cross-linking. Biomaterials. 2002, vol. 23, no. 4, pp. 12051212.

[6] CAMCI-UNAL, G., CUTTICA, D., ANNABI, N., et al. Synthesis and characterization of hybrid hyaluronic acidgelatin hydrogels. Biomacromolecules. 2013, vol. 14, no. 4, pp. 1085-1092.

[7] VANDERHOOFT JL, ALCOUTLABI M, MAGDA JJ, PRESTWICH GD. Rheological Properties of Cross-Linked Hyaluronan-Gelatin Hydrogels for Tissue Engineering. [onlne]. 2009, vol.9, no. 1, pp. 20-8. [Accessed in October 2020] .Available from: https://www.ncbi.nlm.nih.gov/pmc/articles/PMC2711643/.

[8] FRASER, J.R., LAURENT, T.C., LAURENT, U.B. Hyaluronan: its nature, distribution, functions and turnover. Journal of Internal Medicine. 1997, vol. 242, no. 1, pp. 27-33.

[9] KUO, J.W., SWANN, D.A., PRESTWICH, G.D. Chemical modification of hyaluronic acid by carbodiimides. Bioconjugate Chemistry. 1991, vol. 2, no. 4, pp. 232-241.

[10] ARABI, S.H., AGHELNEJAD, B., SCHWIEGER, C., et al. Serum albumin hydrogels in broad pH and temperature ranges: characterization of their self-assembled structures and nanoscopic and macroscopic properties. Biomaterials Science. 2018, vol. 6, no. 3, pp. 478-492.

[11] AKDOGAN, Y., REICHENWALLNER, J., HINDERBERGER, D. Evidence for Water-Tuned Structural Differences in Proteins: An Approach Emphasizing Variations in Local Hydrophilicity. PLOS ONE. 2012, vol. 7, no. 9, p. e45681. 
[12] NEČAS, D., KLAPETEK, P. Gwyddion: an open-source software for SPM data analysis. Open Physics. 2012, vol. 10, no. 1, pp. 181-188.

[13] SCHNEIDER, C.A., RASBAND, W.S., ELICEIRI, K.W. NIH Image to ImageJ: 25 years of image analysis. Nature Methods. 2012, vol. 9, no. 7, pp. 671-675.

[14] HARICH, R., BLYTHE, T.W., HERMES, M., et al. Gravitational collapse of depletion-induced colloidal gels. Soft Matter. 2016, vol. 12, no. 19, pp. 4300-4308.

[15] JPK Instruments. JPK Application Note: Determining the elastic modulus of biological samples using atomic force microscopy.

[16] VREEKER, R., LI, L., FANG, Y., et al. Drying and Rehydration of Calcium Alginate Gels. Food Biophysics. 2008, vol. 3, no. 4, pp. 361-369.

[17] COWMAN, M.K., SCHMIDT, T.A., RAGHAVAN, P., et al. Viscoelastic Properties of Hyaluronan in Physiological Conditions. F1000Research. 2015, vol. 4, p. 622.

[18] GUPTA, M., SARANGI, B.R., DESCHAMPS, J., et al. Adaptive rheology and ordering of cell cytoskeleton govern matrix rigidity sensing. Nature Communications. 2015, vol. 6, no. 1, p. 7525. 